\title{
On a Rigid Body Subject to Point-Plane Constraints
}

\author{
Charles W. Wampler \\ General Motors R\&D Center \\ Mail Code 480-106-359 \\ 30500 Mound Road \\ Warren, MI 48090-9055 \\ E-mail: charles.w.wampler@gm.com
}

Paper MD-05-1167

July 19, 2005

\begin{abstract}
This paper investigates the location of a rigid body such that $N$ specified points of the body lie on $N$ given planes in space. Variants of this problem arise in kinematics, metrology, and computer vision, including some, such as the motion of a spherical four-bar, that are not at first glance point-plane contact problems. The case $N=6$, the minimum number to fully constrain the body, is of special interest: we give an eigenvalue method for finding all solutions, which may number up to eight. For $N \geq 7$ there are, in general, no solutions, but if the constraints are compatible and not degenerate, we show how to find the unique solution by a linear least-squares method. For $N \leq 5$, the body is underconstrained, having in general $6-N$ degrees of freedom; we determine the degree of the general motion for each case. We also examine the workspace of a particular three-degree-of-freedom parallel-link tripod mechanism.
\end{abstract}

\section{Introduction}

We consider the class of problems in which $N$ points of a rigid body are constrained to lie on $N$ known planes. Such problems arise in many guises in the kinematics of mechanisms, in metrology, and in computer vision. Some geometric constraints not initially formulated as point-plane contacts can be easily seen to be equivalent to a collection of such contacts: plane-plane contact is equivalent to constraining three non-collinear points of one body to lie on the same plane of the other, and similarly, line-plane contact can be modeled as two points on a plane. A spherical joint is equivalent to constraining a single point 
to lie on three non-parallel planes, and equivalences exist for other common joints as well. In vision, assuming a pinhole camera model, the position of a feature point in the image plane constrains the corresponding point of the body to lie on a line, a situation equivalent to constraining that point to lie on two known planes. Similarly, the detection of a feature line as an edge in the image plane constrains all points of that feature line on the body to lie on a known plane in space, that is, it is equivalent to a line-plane contact. In metrology, it is common to measure the center point of a sphere touching a planar surface, which is equivalent to constraining an offset plane of the surface to coincide with the measured point. From these observations, it appears that an understanding of multiple simultaneous point-plane constraints has wide applicability.

This paper is an extension of [1], which considered $N \geq 6$ general point-plane constraints. Here, a substantial extension is the treatment of cases with $N \leq 5$ constraints. We use continuation techniques from numerical algebraic geometry to determine the degree of the workspace as an algebraic set and to test if it can be factored into more than one irreducible piece. We show how this applies to a three-degree-of-freedom parallel-link tripod mechanism.

For $N=6$ general point-plane constraints, the body is exactly constrained. We will show that there are at most eight locations of the body meeting all the constraints and give an algorithm for computing them as an $8 \times 8$ eigenvalue problem.

For more than six point-plane constraints, if the data are consistent and the arrangement of points and planes not degenerate, the problem has a unique answer. We show how to find it by solving a linear system of equations, with successively simpler formulations applying in the cases of $N=7,8 \leq N \leq 11$, and $N \geq 12$. In the likely case that the data contains error, there will be no exact answer, but one may use linear least squares to find an approximate one.

\section{Motivating Examples}

To make the discussion more concrete, consider the important case of $N=6$, illustrated in Fig.1. This amounts to the two equivalent problems:

1. determine the location of a rigid body, $\mathbb{B}$, relative to ground, $\mathbb{G}$, by measuring six planes in $\mathbb{G}$, each meeting a known point of $\mathbb{B}$, and

2. solve the forward kinematics of a $6 E S$ parallel-link robot, where " $E$ " denotes a plane joint and " $S$ " denotes a spherical joint.

If one can solve these problems, then by kinematic inversion, that is, by reversing the roles of $\mathbb{G}$ and $\mathbb{B}$, one can also:

3. determine the location of a rigid body, $\mathbb{B}$, relative to ground, $\mathbb{G}$, by measuring six points in $\mathbb{G}$, each meeting a known plane of $\mathbb{B}$, and

4. solve the forward kinematics of a $6 S E$ parallel-link robot. 


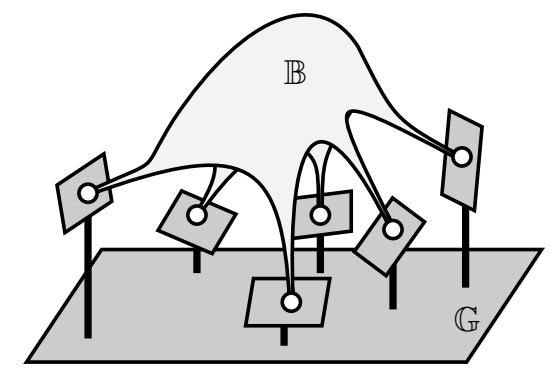

Figure 1: Schematic of locating six points on six planes.

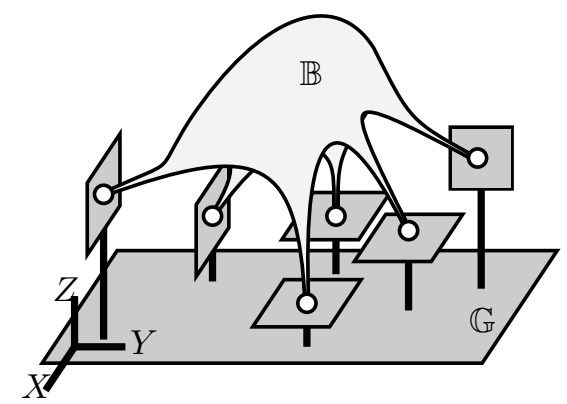

Figure 2: Schematic of 3-2-1 locating.

These problems generally have multiple solutions; that is, there exists more than one location of $\mathbb{B}$ where all six points lie on their respective planes.

In metrology, it is common to locate a rigid body in space by a so-called " 3 2-1" locating scheme, illustrated in Fig.2, in which the $Z$-coordinates of three points, the $Y$-coordinates of two points, and the $X$-coordinate of one point are measured. Since measuring the $X$-coordinate of a point is the same as measuring which plane perpendicular to the $X$-axis meets that point, it is clear that 3-21 locating is a special case of Problem (1) above. Problem (3) arises when a coordinate measuring machine determines the coordinates of a point on a planar surface, such as by touching the surface with a probe or by optically measuring a laser spot projected onto the surface. Note that if the probe is a sphere, then the center of the sphere lies on a plane parallel to the contact plane, offset by the radius of the sphere, so this case is also included in the present analysis.

The $6 E S$ forward kinematics problem concerns any robot mechanism that has unmeasured plane and sphere joints in series on each of six legs. The device can have any number of other joints, as long as their motions are measured. For the purposes of determining the relative locations of all the links of a robot, any measured joint can be temporarily replaced by a rigid connection. In this manner, it can be seen that the forward kinematics of a variety of robot topologies reduce to analyzing the $6 E S$ structure. 


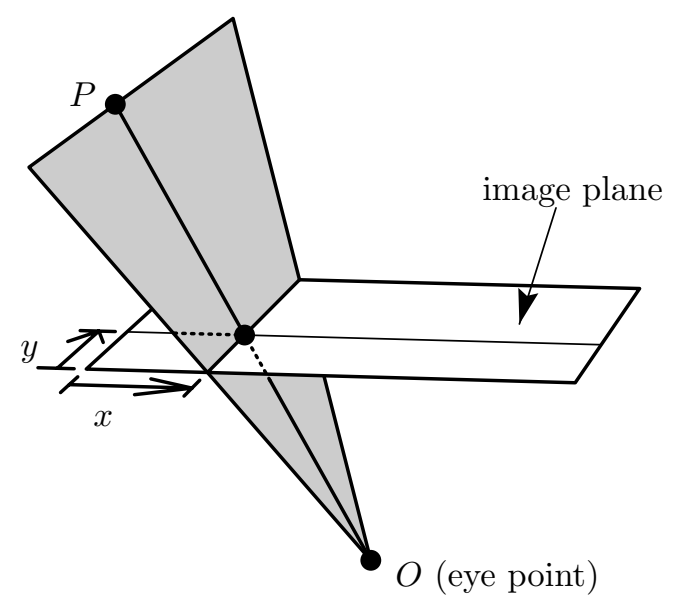

Figure 3: Viewing a feature point $P$ with a pinhole camera.

The $6 E S$ forward kinematic problem is related to the well-studied $6 S S$ forward kinematics problem associated to Stewart-Gough platforms. In the $6 S S$ problem, six points of a body are constrained to fall on known spheres. If we consider a plane as the limit of a sphere whose center goes to infinity, the $6 E S$ structure is a special case of the $6 S S$ structure. It is well-known that the $6 S S$ structure has 40 solutions $[2,3,4,5,6,7]$, which sets an upper bound on the number of solutions that the $6 E S$ structure may have. A somewhat sharper result appears in Theorem 5.4 of [8], based directly on a result from [2], which implies that the $6 E S$ problem can have no more than 20 feasible isolated solutions. We will see that the $6 E S$ problem is actually much simpler than this: it has at most 8 isolated solutions.

Multiple point-plane constraints also arise in machine vision. In particular, for monocular vision with a pinhole camera, one has two point-plane constraints for each feature point of a body detected in the image, as illustrated in Figure 3. We may model this as one plane for the $x$-coordinate of the image point and one for the $y$-coordinate. For $k$ feature points, we have $N=2 k$ constraints, so three points exactly constrain the body, another instance of the $6 E S$ problem.

A body subject to $1 \leq N \leq 5$ point-plane constraints retains, in general, $6-N$ degrees of freedom of motion. This is relevant to the characterisation of the workspace of three-degree-of-freedom parallel-link tripod mechanisms in which each of three legs imposes a point-plane constraint on the moving platform. The resulting constraints are illustrated in Figure 4. This type of arrangement has received considerable attention; for example, a recent issue of this Journal contains three papers on it: calibration in [9], the enumeration of equivalent mechanisms in [10], and dynamics in [11]. We refer to these as a starting point for accessing the literature on this particular mechanism. We study here the properties of its workspace imposed by the point-plane constraints, without re- 


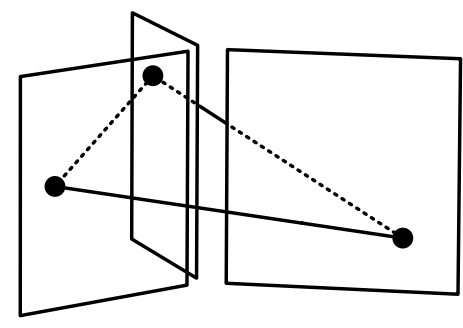

Figure 4: Geometric constraints of the tripod mechanism. The triangle is free to move subject to each vertex remaining on a given plane.

gard for the means by which the points are forced to move in these planes. Most commonly, each leg is a PRS serial linkage, with the prismatic joint actuated and the rotational and spherical joints passive, but many alternatives are enumerated in [10].

Finally, consider a spherical mechanism that constrains one point of body $\mathbb{B}$ to coincide with a given point $\mathcal{O}$ in the ground link, $\mathbb{G}$. This constraint may be implemented directly as a spherical joint, but it may also arise as a consequence of multiple rotational joints whose joint axes intersect at $\mathcal{O}$. Examples include the spherical four bar and spherical parallel-link wrist mechanisms, such as a tripod mechanism with $R R R$ legs whose axes all intersect. The spherical constraint is equivalent to constraining a point of $\mathbb{B}$ to lie on three independent planes intersecting at $\mathcal{O}$. All other points of $\mathbb{B}$ must travel on concentric spheres. For a spherical four-bar, two additional points of $\mathbb{B}$ are constrained to travel on circles whose axes intersect $\mathcal{O}$. Since each point is already constrained to a sphere, the circle constraints are equivalent to intersecting a plane with that sphere. In this way, the motion of $\mathbb{B}$ can be modeled as a system of five point-plane constraints. Similarly, a spherical pentad structure, which has three additional points of $\mathbb{B}$ constrained to circles, can be modeled by six point-plane constraints, and hence, the eight solutions of the pentad spherical structure can be calculated using the algorithm given here for $N=6$, although the formulation in [12] is somewhat more direct.

\section{The Problem}

We are given $N$ points $\mathbf{x}_{i} \in \mathbb{R}^{3}, i=1, \ldots, N$, of a rigid body $\mathbb{B}$ and $N$ planes $\left[\mathbf{n}_{i}, d_{i}\right] \in \mathbb{P}^{3}, i=1, \ldots, N$, in a ground coordinate system, $\mathbb{G}$, where the equation for the $i$-th plane is of the form

$$
\mathbf{n}_{i}^{T} \mathbf{X}+d_{i}=0 .
$$

Here, $\mathbf{X} \in \mathbb{R}^{3}$ is the coordinate vector of a point in $\mathbb{G}, \mathbf{n}_{i}$ is a vector normal to the plane and, if $\mathbf{n}_{i}$ is unit length, $d_{i}$ is the signed distance of the plane from the origin. The notation $\left[\mathbf{n}_{i}, d_{i}\right]$ indicates that scaling $\mathbf{n}_{i}$ and $d_{i}$ by a common, 
nonzero factor does not change the plane. The problem is to determine all ways of positioning body $\mathbb{B}$ in space such that each point $\mathbf{x}_{i}$ lies on plane $\left[\mathbf{n}_{i}, d_{i}\right]$.

A rigid body displacement can be described by a $3 \times 3$ rotation matrix $\mathbf{R} \in$ $S O(3)$ and a translation vector $\mathbf{t} \in \mathbb{R}^{3}$, whereby point $\mathbf{x}_{i}$ in the body coordinates of $\mathbb{B}$ is displaced to point $\mathbf{X}_{i}$ in $\mathbb{G}$ according to the transformation $\mathbf{X}_{i}=\mathbf{R} \mathbf{x}_{i}+\mathbf{t}$. It is useful to generalize this to include scalars $s_{i}, i=1, \ldots, N$, and write

$$
\mathbf{X}_{i}=\mathbf{R} \mathbf{x}_{i}+s_{i} \mathbf{t}
$$

In this way, we may set $s_{i}=1$ to constrain point $\mathbf{x}_{i}$, or instead, we may set $s_{i}=0$ to constrain the direction of vector $\mathbf{x}_{i}$ without regard to its position.

Accordingly, our objective is to find all $(\mathbf{R}, \mathbf{t})$ satisfying the $N$ equations

$$
f_{i}(\mathbf{R}, \mathbf{t})=\mathbf{n}_{i}^{T}\left(\mathbf{R x}_{i}+s_{i} \mathbf{t}\right)+d_{i}=0, \quad i=1, \ldots, N .
$$

There are several different ways to represent rotations. Whichever we may choose, Eq.(3), is a system of $N$ equations on the six-dimensional space of three rotational and three translational degrees of freedom. The planes $\left[\mathbf{n}_{i}, d_{i}\right]$ and the points $\left(\mathbf{x}_{i}, s_{i}\right)$ are known quantities. For $N=6$ general planes and points, $\mathbb{B}$ will be exactly constrained; that is, there will be only a finite number of isolated locations that satisfy Eq.(3).

\section{Representing Rotations}

Before proceeding further, it is useful to consider how best to represent rotations. The main candidates are: rotation matrices, Euler angles, Cayley's formula, Rodrigues parameters, matrix exponentials and quaternions. While descriptions of these are widely available (a good summary can be found in [13, Ch.5], for example), we will review them here to examine their suitability for treating point-plane constraint problems. The last four of these are intimately related and give essentially the same formula, as we shall see below.

Direct representation of a $3 \times 3$ rotation matrix by its nine entries requires the inclusion of the quadratic orthogonality conditions $\mathbf{R}^{T} \mathbf{R}=\mathbf{I}$, where $\mathbf{I}$ is the $3 \times 3$ identity matrix, and the condition $\operatorname{det} \mathbf{R}=1$ to rule out reflections. Lazard [2] gives a Gröbner basis for this set polynomial relations, showing that it is dimension 3 and degree 8 . From this, one may conclude that three general linear equations on the elements of $\mathbf{R}$ have 8 isolated solutions, which establishes a limit on the number of solutions for the general case of $N=6$. However, it is not immediately apparent how to convert this observation into a numerical method for computing the solutions.

Representations by Euler angles, that is, by expressing $\mathbf{R}$ as the product of three successive simple rotations, are familiar to many, but they are not convenient for the task at hand. This is because the elements of $\mathbf{R}$ are cubic functions of sine and cosine of the angles, with the addition of the quadratic trigonometric identity $\sin ^{2} \theta+\cos ^{2} \theta=1$ for each of the angles involved. Consequently, we will not give further consideration to such representations. 
In contrast to the foregoing alternatives, a representation of rotation in terms of quaternions does not introduce additional relations. A quaternion is a 4vector $\mathbf{q}=\left(q_{0}, q_{1}, q_{2}, q_{3}\right)$, which can also be written as a scalar, $q_{0}$, paired with a three-vector, $\hat{\mathbf{q}}$, as $\mathbf{q}=\left(q_{0}, \hat{\mathbf{q}}\right)$. Quaternion multiplication can be conveniently written using this latter form and the vector dot and cross product operators as

$$
\left(a_{0}, \mathbf{a}\right) *\left(b_{0}, \mathbf{b}\right)=\left(a_{0} b_{0}-\mathbf{a} \cdot \mathbf{b}, a_{0} \mathbf{b}+b_{0} \mathbf{a}+\mathbf{a} \times \mathbf{b}\right) .
$$

The operation of quaternion conjugation is written $\left(q_{0}, \hat{\mathbf{q}}\right)^{\prime}=\left(q_{0},-\hat{\mathbf{q}}\right)$. It is a well-known result that, for a pure vector ${ }^{1} \mathbf{a}$, the operation $\mathbf{q} * \mathbf{a} * \mathbf{q}^{\prime} /\left(\mathbf{q} * \mathbf{q}^{\prime}\right)$ yields a pure vector that is a rotation of $\mathbf{a}$. Moreover, every possible rotation has a corresponding quaternion. Expanding this product out, we have the expressions

$$
\begin{aligned}
\mathbf{R a} & =\mathbf{q} * \mathbf{a} * \mathbf{q}^{\prime} /\left(\mathbf{q} * \mathbf{q}^{\prime}\right):=\hat{\mathbf{R}}(\mathbf{q}) \mathbf{a} /\left(\mathbf{q}^{T} \mathbf{q}\right) \\
\hat{\mathbf{R}}(\mathbf{q}) & =\left(\begin{array}{ccc}
q_{0}^{2}+q_{1}^{2}-q_{2}^{2}-q_{3}^{2} & 2\left(-q_{0} q_{3}+q_{1} q_{2}\right) & 2\left(q_{0} q_{2}+q_{1} q_{3}\right) \\
2\left(q_{0} q_{3}+q_{2} q_{1}\right) & q_{0}^{2}-q_{1}^{2}+q_{2}^{2}-q_{3}^{2} & 2\left(-q_{0} q_{1}+q_{2} q_{3}\right) \\
2\left(-q_{0} q_{2}+q_{3} q_{1}\right) & 2\left(q_{0} q_{1}+q_{3} q_{2}\right) & q_{0}^{2}-q_{1}^{2}-q_{2}^{2}+q_{3}^{2}
\end{array}\right) .
\end{aligned}
$$

This expression for $\mathbf{R}$ is homogeneous; that is, $\mathbf{q}$ and $\alpha \mathbf{q}$ give the same rotation for any nonzero scalar $\alpha$. One may often see this formula employed with the restriction of $\mathbf{q}$ to unit magnitude, i.e., $\mathbf{q}^{T} \mathbf{q}=1$. For our purposes, this would merely introduce an extra quadratic relation that would double the number of solutions, since $\mathbf{q}$ and $\mathbf{- q}$ have the same magnitude and give the same rotation. It is better to let $\mathbf{q}$ be a general quaternion and use Eq.(5). Said another way, we take $\mathbf{q}=\left[q_{0}, q_{1}, q_{2}, q_{3}\right] \in \mathbb{P}^{3}$ as a point in projective three space and our equations are homogeneous in $\mathbf{q}$.

Rodrigues parameters and the Cayley formula can both be viewed as dehomogenizations $^{2}$ of the quaternion representation. The Rodrigues form says that a rotation of vector $\mathbf{a}$ through an angle $\theta$ about a unit vector $\mathbf{v}$ is given in terms of the scaled vector $\mathbf{r}=\tan \frac{\theta}{2} \mathbf{v}$ as

$$
\mathbf{R a}=\mathbf{a}+2(\mathbf{r} \times \mathbf{a}+\mathbf{r} \times(\mathbf{r} \times \mathbf{a})) /(1+\mathbf{r} \cdot \mathbf{r}) .
$$

Expanding this expression and comparing to Eq.(6), one may see that they are equivalent with the rescaling $\mathbf{q}=\left(1, q_{1} / q_{0}, q_{2} / q_{0}, q_{3} / q_{0}\right)=\left(1, r_{1}, r_{2}, r_{3}\right)$. Another expression is Cayley's formula, which gives an orthogonal matrix of any size as

$$
\mathbf{R}=(\mathbf{I}-\mathbf{B})^{-1}(\mathbf{I}+\mathbf{B}),
$$

where $\mathbf{B}$ is skew symmetric. When applied in three dimensions with the entries of $\mathbf{B}$ denoted as

$$
\mathbf{B}=\left(\begin{array}{ccc}
0 & -r_{3} & r_{2} \\
r_{3} & 0 & -r_{1} \\
-r_{2} & r_{1} & 0
\end{array}\right)
$$

\footnotetext{
${ }^{1} \mathrm{~A}$ pure vector is a quaternion whose scalar part is zero.

${ }^{2} \mathrm{~A}$ point in projective space is dehomogenized by setting one coordinate, or a linear combination of coordinates, to a constant, thereby removing the freedom to rescale.
} 
Cayley's formula is exactly the same as the Rodrigues formula. The Rodrigues and Cayley forms have a singularity when $\theta=\pi$, for then $\tan \frac{\theta}{2}$ is infinite and, hence, so is $\mathbf{r}$. These rotations are no trouble for the quaternion representation; they correspond to $q_{0}=0$.

Finally, we note that the matrix exponential representation of rotation is again equivalent to the Rodrigues representation. Letting $\mathbf{V}$ be the skew symmetric matrix formed from a unit vector $\mathbf{v}$ in the same pattern as $\mathbf{B}$ is formed from $\mathbf{r}$ in Eq.8 and defining the matrix exponential as $e^{A}=\sum_{i=0}^{\infty} A^{i} / i$ !, one may show that the exponential $e^{\theta \mathbf{V}}$ is a rotation matrix. Using the fact that $\mathbf{V}^{3}=-\mathbf{V}$, one may reduce all cubic and higher powers of $\mathbf{V}$ to $\pm \mathbf{V}$ or $\pm \mathbf{V}^{2}$ and thereby rewrite the rotation as

$$
\mathbf{R}=e^{\theta \mathbf{V}}=\mathbf{I}+\sin \theta \mathbf{V}+(1-\cos \theta) \mathbf{V}^{2} .
$$

Half-angle formulas for sine and cosine convert this to the Rodrigues formula, with $\mathbf{r}=\tan \frac{\theta}{2} \mathbf{v}$ and $\mathbf{B}=\tan \frac{\theta}{2} \mathbf{V}$.

In summary, the quaternion representation leads us directly to a homogeneous polynomial formulation for the rotation matrix. The Rodrigues and Cayley forms are just dehomogenized quaternions. Matrix exponentials expressed as an infinite series are of no use to us in formulating a polynomial system to solve the $N$ plane system, and when converted to polynomials by the skew-symmetric property $\mathbf{V}^{3}=-\mathbf{V}$, we again get the Cayley form. So from this point on, we will only speak of the quaternion formulation.

Using quaternions as in Eq.(5) and clearing $\mathbf{q}^{T} \mathbf{q}$ from the denominator, the point-plane constraints Eq.(3) become for $i=1, \ldots, N$

$$
\begin{aligned}
F_{i}(\mathbf{q}, \mathbf{t}) & =\left[\mathbf{n}_{i}^{T} \hat{\mathbf{R}}(\mathbf{q}) \mathbf{x}_{i}+\mathbf{q}^{T} \mathbf{q} d_{i}\right]+\mathbf{q}^{T} \mathbf{q} s_{i} \mathbf{n}_{i}^{T} \mathbf{t}=0 \\
& =\mathbf{q}^{T} \mathbf{M}_{i} \mathbf{q}+\mathbf{q}^{T} \mathbf{q} \mathbf{N}_{i} \mathbf{t}
\end{aligned}
$$

where $\mathbf{M}_{i}$ is a $4 \times 4$ symmetric matrix of constants that depend on the given data $\mathbf{n}_{i}, \mathbf{x}_{i}, d_{i}$ and where $\mathbf{N}_{i}=s_{i} \mathbf{n}_{i}^{T}$ is a $1 \times 3$ row of constants. Each point-plane constraint is a cubic equation that is quadratic, homogeneous in $\mathbf{q}$ and linear, inhomogeneous in $\mathbf{t}$.

\section{Cases with Free Translation}

Let $\mathbf{N}$ denote the $N \times 3$ matrix whose rows are $\mathbf{N}_{i}$ from Eq.(10). When $\operatorname{rank} \mathbf{N}$ is less than three, $\mathbb{B}$ may translate freely in directions in the right null-space of $\mathbf{N}$. This must happen, of course, if $N<3$, but it may also come about if the normals to the planes do not span three space. If $\operatorname{rank} \mathbf{N}=2$, we can find a nonzero vector $\mathbf{v}$ with $\mathbf{N v}=0$ to describe the free translational direction. Then, the motion of $\mathbb{B}$ may be understood by initially adding the constraint $\mathbf{v}^{T} \mathbf{t}=0$, analyzing the remaining motion of $\mathbb{B}$, and then reintroducing the free translation. The new constraint is a point-plane condition applied to the origin of $\mathbb{B}$, so we may proceed as if the mechanism had $N+1$ point-plane constraints. If $\operatorname{rank} \mathbf{N}$ is one, we work in a similar way, adding two translational constraints, 
respectively. In the case $\operatorname{rank} \mathbf{N}=0$, which occurs only if all the constraints are directional $\left(s_{i}=0\right.$ for all $\left.i\right)$, one may introduce three translational constraints.

If after adding translational constraints, there are a total of six or more constraints, we will treat the problem with the methods for $N \geq 6$ below. Generally, the mechanism will have a finite number of orientations it can attain, and in each of these orientations, it can translate freely within the null space of $\mathbf{N}$. If on the other hand, we still have $N<6$, there are extra degrees of freedom that involve coordinated motion in orientation and translation. We will address this in the next section.

\section{Incompletely Constrained Mechanisms}

For $N \leq 5, \mathbb{B}$ has, in general, $6-N$ degrees of freedom. For any particular mechanism, one could map out its motion in various ways. For a one-degreeof-freedom mechanism, one might create an animation of the motion or plot the paths in space of selected points of $\mathbb{B}$. For two degrees of freedom, points generally sweep out a surface in space, so one might study these surfaces. For higher degrees of freedom, one might plot the motion of points when the orientation is constrained in various ways. One often sees, for example, plots of a manipulator's reachable volume when the orientation is held constant. Because they can be plotted and visualized, the sets swept out by points of $\mathbb{B}$ under various orientation constraints are appealing. They have the shortcoming, however, that the orientational motion and its coupling with the translational motion are only sampled roughly. An alternative is to investigate the entire motion as a $(6-N)$-dimensional subset of the 6 -dimensional space of translation and rotation. Although this set cannot be visualized directly, we can determine some of its properties.

Since the point-plane constraints are algebraic, the curves or surfaces swept out by a point or the entire motion set will all be algebraic sets. Two basic properties of an algebraic set are its degree and the number of irreducible components that it contains. A reducible algebraic set, for example, a collection of lines, consists of more than one irreducible piece, which meet each other in complex space only at singularities. After removing singularities, those sets which remain connected are irreducible.

To compute these properties, it is helpful to first dehomogenize the quaternion representation of rotations by choosing an arbitrary patch on $\mathbb{P}^{3}$ via the linear form $w(\mathbf{q}):=\mathbf{a} \cdot \mathbf{q}$, where $\mathbf{a} \in \mathbb{C}^{4}$ has been chosen generically. The motion for $N$ point-plane constraints is the algebraic set

$$
V_{N}:=\left\{(\mathbf{q}, \mathbf{t}) \in \mathbb{C}^{7} \mid F_{1}(\mathbf{q}, \mathbf{t})=0, \ldots, F_{N}(\mathbf{q}, \mathbf{t})=0, w(\mathbf{q})=1\right\} .
$$

We would like to assess the degree and irreducibility of $V_{N}$ for $N<6$ point-plane constraints. 


\subsection{Determining Degree and Irreducibility}

To determine the degree of an algebraic set of dimension greater than zero (i.e., a "positive dimensional set"), we may use the approach propounded in [14] and explained further in $[15,16]$ of intersecting the set with a generic linear space of commensurate co-dimension. The slice so obtained consists of isolated points, called witness points, whose number is equal to the degree of $V_{N}$. Moreover, the paths swept out by the witness points as the linear slicing space is moved continuously reveal whether or not the set is irreducible. Monodromy loops, formed by moving the slicing space around a closed path and tracking the paths of the witness points, can find witness points that connect without encountering a singularity, thereby showing that they are on the same irreducible component $[16,17]$. When the number of witness points is not too large, or after monodromy has reduced the number of possible groupings, the decomposition into irreducible components can be completed by exhaustive testing of linear traces $[16,18$, $19,20]$. In the problems at hand, it turns out that the number of witness points is never greater than 15 , so exhaustive trace testing is feasible without preprocessing by monodromy.

The idea of linear trace testing is illustrated in Figure 5 for a single quartic equation that factors into two ellipses. When the pair of ellipses are cut by a generic line, four witness points are obtained. The figure has been arranged so that these are all real, although in general, we would take random lines in complex space so as to avoid singularities. When the slicing line is translated parallel to itself, the centroid of a witness set must travel in a straight line. For the entire witness set, the centroid follows line $L_{0}$, but we see that the centroids for the two irreducible pieces also travel on lines, marked as $L_{1}$ and $L_{2}$. An irreducible decomposition may be found by checking all possible subsets of the witness paths to see which centroids follow lines. If it is true only for the entire set of witness paths and not for any of the subsets, we know the algebraic set is irreducible.

\subsection{General Cases $N \leq 5$}

To compute a witness sets for a $k$-dimensional component, we append $k$ linear equations in $(\mathbf{q}, \mathbf{t})$, which we may write in matrix notation as $A \mathbf{q}+B \mathbf{t}+C=0$. The complete set of equations to be solved are

$$
G_{N, k}(\mathbf{q}, \mathbf{t})=\left[\begin{array}{c}
F_{1}(\mathbf{q}, \mathbf{t}), \ldots, F_{N}(\mathbf{q}, \mathbf{t}) \\
\mathbf{a} \cdot \mathbf{q}-1 \\
A \mathbf{q}+B \mathbf{t}+C
\end{array}\right]=0
$$

where the linear slicing equations are given by $A \in \mathbb{C}^{k \times 4}, B \in \mathbb{C}^{k \times 3}$, and $C \in$ $\mathbb{C}^{k \times 1}$, all random complex matrices. When we are considering general pointplane constraints, we expect to find solution components only at dimension $k=6-N$. To be certain, however, we checked also for higher-dimensional solution components, using randomization to square up these systems, as they 


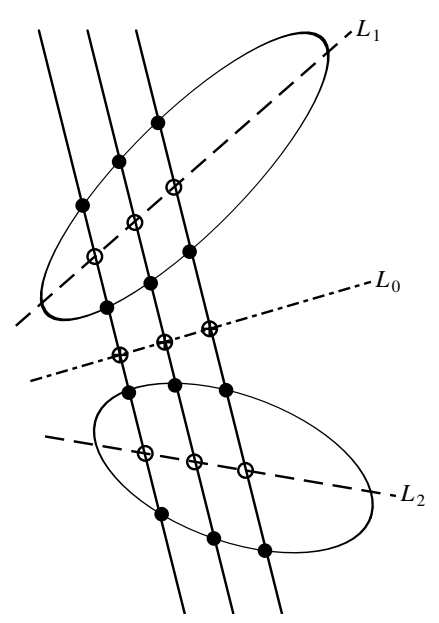

Figure 5: Linear traces. Under parallel translation of the slicing line, the centroid of a witness set must follow a line. This is true for a composite set $\left(L_{0}\right)$ and for each of its irreducible pieces $\left(L_{1}, L_{2}\right)$.

\begin{tabular}{|r|c|c|c|c|c|}
\hline$N$ & 1 & 2 & 3 & 4 & 5 \\
$\operatorname{deg}\left(V_{N}\right)$ & 3 & 7 & 15 & 14 & 12 \\
\hline
\end{tabular}

Table 1: Degree of the motion variety $V_{N}=V\left(F_{1}, \ldots, F_{N}\right)$ for $N \leq 5$.

have more equations than unknowns, see $[14,16]$. No finite higher-dimensional components were present in any case.

For $k=N-6$, we have the same number of equations as variables, and we may solve them with continuation. Doing so for each $N \leq 5$ with randomly generated point-plane constraints gives a witness set for each of the associated motion varieties. The witness points were in every case nonsingular. Their number, given in Table 1, is the degree of the motion set. While these results were obtained numerically, we explain them analytically in Appendix A.

We next compute the witness paths generated as the constants $C$ in the slicing equations are moved linearly, that is, we replace $C$ by $C+C^{\prime} t$ for a random $C^{\prime} \in \mathbb{C}^{k \times 1}$ and vary $t \in[0,1]$. In every case, the linear trace test shows that the motion set is irreducible.

These results are for general point-plane constraints. If the constraints are specially situated, then the degree of $V_{N}$ may decrease, it may factor into subcomponents, or both. We will return to this later when we study the tripod mechanism. 


\section{Completely Constrained Mechanisms}

We consider now cases of $N \geq 6$ in which $\mathbb{B}$ is completely constrained, ignoring degenerate cases in which $\mathbb{B}$ has a positive-dimensional motion. As discussed in Section 5, a mechanism having free translational motions may become nondegenerate for our purposes after the translation is artificially constrained.

Under these assumptions, we have that matrix $\mathbf{N}$ has full rank three. In Eq.(10), the quantity $\hat{\mathbf{t}}:=\mathbf{q}^{T} \mathbf{q t}$ appears linearly, so it can be easily eliminated from the equations, for example, by using Gaussian elimination to get $N-3$ equations of the form

$$
\mathbf{q}^{T} \widetilde{\mathbf{M}}_{i} \mathbf{q}=0, \quad i=1, \ldots, N-3 .
$$

where each $\widetilde{\mathbf{M}}_{i}, i=1, \ldots, N-3$ is a linear combination of the $\mathbf{M}_{i}, i=1, \ldots, N$, from Eq.(10). Once we solve these for $\mathbf{q}$, we can use the results of the Gaussian elimination stage to backsolve uniquely for $\hat{\mathbf{t}}$. Each solution $\mathbf{q}$ with $\mathbf{q}^{T} \mathbf{q} \neq 0$ gives a unique, finite value of translation as $\mathbf{t}=\hat{\mathbf{t}} / \mathbf{q}^{T} \mathbf{q}$; otherwise, the solution is a degenerate one at infinity.

\subsection{Case $N=6$}

For the case $N=6$, we have three quadratics on $\mathbb{P}^{3}$, so by Bézout's Theorem, there are at most $2^{3}=8$ isolated solutions over the complex numbers. If the planes and the points are special, it could happen that the problem simplifies and the number of roots decreases. It could also happen that the problem becomes degenerate, so that the solutions are not isolated but instead form a solution curve or surface. We will not explore these possibilities in this article; we only treat the case where the problem is general and has 8 isolated roots. Consequently, what remains to be done is to apply any of several existing methods for solving three quadratic equations. The next section addresses this issue.

\subsubsection{Solving Three Quadratics}

The solution of three general quadratics is a classical problem with many possible avenues of attack. A common approach is to seek an elimination procedure that produces a single octic polynomial in one variable. An early result of this sort is the method of Dixon [21]. A more modern approach is to compute a Gröbner basis for the system of polynomials [22] and then derive an eigenvalue problem from it $[23,24]$. Since one of the best ways to solve a polynomial of degree less than about 50 in one variable is to solve an eigenvalue problem for its companion matrix [25], we take the approach of forming an eigenproblem in the most direct, numerically stable way that we can find. The detailed derivation of the following algorithm is given in [1], including an interpretation of it as a "numerical Gröbner basis." The approach is similar to the one used in [26, 27].

Assume we have three quadratics, $p_{i}(x, y, z), i=1,2,3$ and we wish to find their common zeros. There are ten possible monomials appearing, namely $\left\{1, x, y, z, x^{2}, x y, x z, y^{2}, y z, z^{2}\right\}$. Do the following: 
1. Multiply each $p_{i}$ by the nine monomials

$$
\left\{1, x, y, z, x^{2}, x y, x z, y^{2}, y z\right\}
$$

to produce an expanded set of 27 polynomials. This expanded set of polynomials has exactly the same zero set as the original three, as it consists of those three and multiples of them.

2. The expanded system has 34 possible monomials, consisting of all monomials of degree 4 or less, except $z^{4}$. List these in a column vector, $\mathbf{m}$, and order them as $\mathbf{m}=\left\{\mathbf{m}_{1}, \mathbf{m}_{2}\right\}$ so that $\mathbf{m}_{2}$ is the "normal set" of 8 monomials $\left\{x z, z, x y, y, x^{3}, x^{2}, x, 1\right\}$ and $\mathbf{m}_{1}$ is the other 26 monomials. Write the system in the form $A \mathbf{m}=A_{1} \mathbf{m}_{1}+A_{2} \mathbf{m}_{2}=0$, where $A$ is a sparse $27 \times 34$ matrix. Matrix $A$ has just ten nonzero entries in each row; these are copies of the coefficients in the original polynomials.

3. For general quadrics $p_{1}, p_{2}, p_{3}$, it turns out that $\operatorname{rank} A_{1}=26$. If for a particular set of quadrics the rank is lower, the method fails. If this is due to having isolated solutions at infinity, then we can get around the problem by using a new projective patch, as we discuss further below. If this is due to the presence of a positive dimensional solution set, an alternative approach must be sought. We do not address that here.

4. Since $A_{1}$ is full column rank, we may solve for $\mathbf{m}_{1}$ in terms of $\mathbf{m}_{2}$ as $\mathbf{m}_{1}=$ $-A_{1}^{\dagger} A_{2} \mathbf{m}_{2}$, where $A_{1}^{\dagger}$ is the pseudoinverse of $A_{1}$. This is a convenient way of writing the solution, but the actual computation can be done with Gaussian elimination or any other stable linear solution method.

5. Choose random $\alpha_{0}, \alpha_{1}, \alpha_{2}, \alpha_{3}$ and let $\lambda(x, y, z)=\alpha_{0}+\alpha_{1} x+\alpha_{2} y+\alpha_{3} z$. When fully expanded, the monomials appearing in $\lambda(x, y, z) \mathbf{m}_{2}$ are all in $\mathbf{m}$, that is, $\lambda(x, y, z) \mathbf{m}_{2}=B \mathbf{m}=B_{1} \mathbf{m}_{1}+B_{2} \mathbf{m}_{2}$. Matrix $B$ is a sparse $8 \times 34$ matrix with four nonzero entries per row, these being copies of $\alpha_{0}, \alpha_{1}, \alpha_{2}, \alpha_{3}$ appropriately arranged.

6. Using the expression derived in Step 4 , eliminate $\mathbf{m}_{1}$ to obtain the expression $\lambda \mathbf{m}_{2}=\left(-B_{1} A_{1}^{\dagger} A_{2}+B_{2}\right) \mathbf{m}_{2}$. This is an eigenvalue problem, with eigenvalue $\lambda$ and eigenvector $\mathbf{m}_{2}$.

7. Solve the eigenvalue-eigenvector problem using a standard routine from linear algebra. Eigenvectors have arbitrary scaling, but the proper scaling is easily determined, as one of the entries in $\mathbf{m}_{2}$ is defined to be 1 . After rescaling to make this so, we may read off $x, y, z$ where they appear in $\mathbf{m}_{2}$.

\subsection{A Word on Homogenization}

In the above presentation, we discussed solving three inhomogeneous quadratics in three variables. In contrast, the system of Eq.12 consists of homogeneous equations in four variables. One approach is to simply dehomogenize the equations by setting $q_{0}=1$, which is equivalent to employing the Rodrigues form. 
This is undesirable if there is a possibility for one of the solutions to be a rotation of $180^{\circ}$.

Such trouble is easily circumvented. Before initiating the solution of the three-quadratic system, we may make a change of coordinates by letting $\mathbf{q}=P \overline{\mathbf{q}}$ for some nonsingular matrix $P$ and solve the transformed equations

$$
\overline{\mathbf{q}}^{T} \overline{\mathbf{M}}_{i} \overline{\mathbf{q}}:=\overline{\mathbf{q}}^{T}\left(P^{T} \widetilde{\mathbf{M}}_{i} P\right) \overline{\mathbf{q}}=0, \quad i=1, \ldots, N-3 .
$$

We now dehomogenize in the new variables via the substitution $\overline{\mathbf{q}}=(1, x, y, z)$. If we choose $P$ at random, the dehomogenization operation sets a random combination of $q_{0}, q_{1}, q_{2}, q_{3}$ to one. There is a zero probability that the combination so selected will be zero for the solutions of the problem at hand. Sometimes called a "projective transformation," this maneuver has proven very effective in numerical work and is regularly employed in polynomial continuation codes to avoid degeneracies [28]. If by rare chance we pick an inauspicious dehomogenization, we may pick a different $P$ and try again. As reported in [1], numerical experiments on 1000 randomly generated examples of case $N=6$ gave just 15 trials whose numerical error was greater than $10^{-6}$, but with a second run of the algorithm (with a new $P$ ), no result had an error greater than $10^{-7}$. These trials, which were computed in Matlab using double precision on a $2 \mathrm{Ghz}$ Xeon processor under Windows, averaged $6 \mathrm{msec}$ per trial, including back-solving for $\mathbf{t}$.

\section{$8 \quad N \geq 7$ Points on $N$ Planes}

For $N \geq 7$ general points and planes, the system of equations will be incompatible, having no solutions. But if the coefficients derive from a compatible situation, for example, from measuring points on $N$ different planar faces of a real object, then the equations should be compatible, within measurement error. The extra constraints will, in general, exclude all but one of the eight solutions we would find for, say, the first six constraints. Since the answer is unique, we may expect that it can be found from solving a linear system of equations. This is indeed possible. Let us again start with Eq.(13), considered as $N-3$ quadratics in $x, y, z$. The most efficient formulation changes as $N$ increases, as follows.

- For $N=7$, we proceed just as in the $N=6$ case, but now the augmented system $A \mathbf{m}=0$ has $9 \cdot 4=36$ equations in 34 monomials. For compatible equations, $A$ has exactly rank 33 and $\mathbf{m}$ is its unique null vector. Whether $A$ is compatible or not, its singular value decomposition can be used to find the unique vector $\mathbf{m}^{*}$ which minimizes the two-norm $\|A \mathbf{m}\| /\|\mathbf{m}\|$. We scale it so that monomial 1 has the correct value and then read off $x, y, z$.

- For $8 \leq N \leq 11$, it is sufficient to generate the augmented system by multiplying the quadratics by just the monomials $\{1, x, y, z\}$. One obtains 
$4(N-3)$ equations in the 20 monomials of degree 3 or less in three variables which can be solved by linear methods.

- For $N \geq 12$, we have at least nine equations and only 10 monomials, one of which is 1 . For $N=12$, we solve the equations directly as a linear system, and for $N>12$, we proceed using linear least squares.

In any of these cases, when the data are not perfect, one cannot expect the monomials in $\mathbf{m}^{*}$ to exactly obey the appropriate interrelationships. For example, $x$ and $x^{2}$ as elements of $\mathbf{m}^{*}$ will not exactly satisfy $x \cdot x=x^{2}$. Nevertheless, the agreement should be close, and we can extract an approximate solution from the linear monomials in $\mathbf{m}^{*}$. If desired, this can be used as the initial guess for iterative refinement of the estimate of the body's location. The refinement can be posed as a maximum likelihood estimation problem taking into account the statistical properties of the measurements. The procedures above can be viewed as a way to generate a good initial guess for the refinement.

Numerical experiments verify that these methods are valid, and in particular, that the linear systems formed in the algorithms have the required rank conditions for general point-plane constraints. The implementations run progressively faster up to $N=12$, averaging just $2 \mathrm{msec}$ in that case.

\section{Special Cases}

So far, we have considered general point-plane constraints. In many practical situations, the points and planes are arranged in special configurations. A few such arrangements are considered next.

\section{$9.1 \quad 3-2-1$ Locating}

Figure 2 illustrates the common 3-2-1 locating scheme. This special case has only four solutions instead of eight. This causes no special trouble for the $N=6$ algorithm: in addition to the desired solutions, the algorithm gives four solutions with tiny values of $\mathbf{q}^{T} \mathbf{q}$. These are mathematical figments of the formulation having no physical meaning and can be considered to be numerical approximations of solutions "at infinity.". Indeed, if one carries on with backsolving, these give large values of $\mathbf{t}$. If one wishes to solve 3-2-1 problems frequently, it might be better to design an algorithm especially for that purpose in a manner that generates only the four finite solutions at the outset.

\subsection{Monocular Vision}

As illustrated in Figure 3, each feature point found in the image is equivalent to two point-plane constraints. A line segment feature is also equivalent to two point-plane constraints. With 3 such features, we have an instance of the $N=6$ problem, and there are at most 8 solutions. For three point features, these appear in four symmetric pairs, related by reflection through the eye-point. 
These are easiest to spot if the origin of coordinates of the body is in the plane of the three points, for then the translation $\mathbf{t}$ appears in sign-symmetric pairs. As the object must be in front of the camera (or eye), at most four solutions are feasible. The symmetry can be used to reformulate the problem as a degree four system.

For four or more features, there will be a unique answer, computable using the algorithms specified above for $N \geq 8$. For six or more features, we already have the simplest case of $N \geq 12$.

\subsection{Tripod Mechanism}

Consider next the case of the tripod mechanism, whose point-plane constraints are illustrated in Figure 4. We assume that the planes all intersect in a common line and that they are spaced evenly at $120^{\circ}$ intervals. Moreover, the three points of the moving body are assumed to form an equilateral triangle.

Let us choose a coordinate system in the ground with the $z$-axis aligned with the line of intersection of the planes. Clearly, the body has a free translation along this direction, so we analyze the rest of the workspace motion by adding a fourth constraint $\left[\begin{array}{lll}0 & 0 & 1\end{array}\right] \cdot \mathbf{t}=0$. From the analysis above for general $N=4$ point-plane constraints, we know that the workspace is an algebraic set of degree at most 14 .

To investigate the workspace, we proceed as in Section 6 by slicing it with two hyperplanes in $\mathbf{C}^{7}$ and then translating the slice to compute linear traces. The result is that the workspace is degree 14, but it factors into two degree 7 components. Examination of the witness points reveals that on one component, the $z$ element of $\mathbf{q}$ is zero, while on the other, the scalar part of $\mathbf{q}$ is zero. In other words, the first component consists of rotations about a vector in the $(x, y)$-plane, while the second component is the composition of such a rotation with a $180^{\circ}$ rotation around the $z$ direction. Notice that if we had formulated the problem using Rodriques parameters, instead of using a random patch on $\mathbb{P}^{3}$, the second component would be trouble.

In complex space without limits on the extent of the constraint planes, the two components may meet, but only in singularities. In practical construction, where the planes are limited and where, as always, only real solutions matter, the device will only operate in one of the two components. With this restriction, we may say that the workspace of the device is degree 7 .

\subsection{Tripod inverse positions}

We may further investigate the tripod by considering its use as a positioning robot. Select a point, say $\mathbf{x}_{E}$, where $E$ is for "endpoint," of the moving body $\mathbb{B}$ and use the three degrees of freedom of the device to position that point in three-space. Denoting the specified position as $\left(p_{x}, p_{y}, p_{z}\right)$, this is equivalent to constraining $\left[\begin{array}{lll}1 & 0 & 0\end{array}\right] \cdot \mathbf{X}_{E}=p_{x},\left[\begin{array}{lll}0 & 1 & 0\end{array}\right] \cdot \mathbf{X}_{E}=p_{y},\left[\begin{array}{lll}0 & 0 & 1\end{array}\right] \cdot \mathbf{X}_{E}=p_{z}$, where $\mathbf{X}_{E}$ is the position of $\mathbf{x}_{E}$ in the ground coordinate system. In other words, we have a 
case of $N=6$ and we expect 8 solutions. By symmetry, we expect four each of these to be on the two workspace components.

This can be understood another way. First, restrict the origin of $\mathbb{B}$ to $z=0$. Now, the mechanism has just two degrees of freedom and $\mathbf{x}_{E}$ sweeps out a surface $S_{E}$ in three-space as the device moves through its range of motion. If we find the configurations such that $\mathbf{x}_{E}$ meets the vertical line $\left[\begin{array}{ll}1 & 0\end{array}\right] \cdot \mathbf{X}_{E}=p_{x}$, $\left[\begin{array}{lll}0 & 1 & 0\end{array}\right] \cdot \mathbf{X}_{E}=p_{y}$, we can then translate along the $z$ direction to attain the desired position. In general, the surface $S_{E}$ and the vertical line intersect in four points (over the complexes) on each of the two workspace components. If we subsequently translate the vertical line in a vertical plane and compute traces, we find that these two sets are each irreducible.

The upshot is that if we restrict the mechanism to just one workspace component, say the one where the $z$ element of $\mathbf{q}$ is zero, then it is possible to formulate the inverse position problem as a degree four eigenvalue problem. Just formulate the quadratics as in Eq.(12) but set the last element of $\mathbf{q}$ to zero. We have 3 quadrics in just two variables, but they are linearly dependent, so the total degree is just $2^{2}$. Solving the pair of quadrics gives the four desired roots. A similar procedure works for the four solutions on the other workspace component.

\subsection{Pointing plus $z$-translate}

We might prefer to use the tripod as an orientational mechanism, in which we orient the normal direction of the plane of $\mathbb{B}$ and control the position of its centroid in the $z$ direction. Suppose we pick $\mathbf{x}_{E}$ as $(0,0,1)$ in body coordinates. Then, we want to specify $\left(\mathbf{X}_{E}-\mathbf{t}\right)$ in ground coordinates as a given unit vector along with $\left[\begin{array}{lll}0 & 0 & 1\end{array}\right] \cdot \mathbf{t}=0$. The direction constraints may be imposed using $s_{i}=0, i=4,5,6$, as allowed in Eq.(2), which with the $z=0$ constraint and the three point-plane pairs of the device itself makes a total of seven pointplane constraints. For seven general compatible constraints, we expect a unique solution, but these constraints are not general. There is, in fact, one solution for each of the two workspace components. Restricting to one of the components, the solution is unique, and there should be a linear formulation for it. Lacking this, we may solve it by applying the procedure for the positioning case to six of the constraints (i.e., ignoring one of the pointing coordinate directions) and then picking out the one solution of the four that satisfies the final constraint.

\section{Conclusion}

We have shown how to efficiently and reliably solve the generalized locating problem of placing $N$ given points of a rigid body on $N$ known planes, a problem that is relevant to metrology, monocular vision, and to certain parallel-link platform robots. The case $N=6$ is reducible to a set of three quadratic equations having 8 solutions in general, and we give an algorithm to compute them via eigenvalue routines. For $N \geq 7$, we show how to reduce the problem to 
solving a set of linear equations. For $N \leq 5$, we use continuation methods to reveal the degree and irreducibility of the workspace as an algebraic set. This approach is particularly revealing when applied to the tripod mechanism, where it shows that the workspace can be factored into two degree-seven pieces. We also discuss the special situations arising when the tripod mechanism is applied to just position or to pointing a vector in a given direction.

\section{References}

[1] C.W. Wampler, "Locating $N$ points of a rigid body on $N$ given planes," Proc. ASME Design Engineering Technical Conf. (CDROM), Salt Lake City, 2004.

[2] D. Lazard, "On the representation of rigid-body motions and its application to generalized platform manipulators," In Computational Kinematics, J. Angeles, P. Kovacs, G. Hommel (eds.), pp.175-182, Kluwer, 1993.

[3] F. Ronga and T. Vust, "Stewart platforms without computer?" Proc. Conf. Real Analytic and Algebraic Geometry, pp.197-212, Trento, 1992.

[4] B. Mourrain, "The 40 generic positions of a parallel robot," Proc. ISSAC, M. Bronstein, Ed., pp. 173-182, 1993.

[5] M. Raghavan, "The Stewart platform of general geometry has 40 configurations," ASME J. Mech. Design, 115:277-282, 1993.

[6] M.L. Husty, "An algorithm for solving the direct kinematics of general Stewart-Gough platforms", Mech. Mach. Theory, 31:4:365-380, 1996.

[7] C.W. Wampler, "Forward displacement analysis of general six-in-parallel SPS (Stewart) platform manipulators using soma coordinates," Mech. Mach. Theory, 31:3:331-337, 1996.

[8] X.S. Gao, D. Lei, Q. Liao and G.-F. Zhang, "Generalized Stewart-Gough platforms and their direct kinematics," IEEE Trans. Robotics, 21:2:141$151,2005$.

[9] M. Verner, F. Xi and C. Mechefske, "Optimal calibration of parallel kinematic machines," ASME J. Mech. Design, 127:1:62-69, 2005.

[10] P. Huynh and J.M. Hervé, "Equivalent kinematic chains of three degreeof-freedom tripod mechanisms with planar spherical bonds," ASME J. Mech. Design, 127:1:95-102, 2005.

[11] F. Xi, O. Angelico, and R. Sinatra, "Tripod dynamics and its inertial effect," ASME J. Mech. Design, 127:1:144-149, 2005.

[12] C.W. Wampler, "Displacement analysis of spherical mechanisms having three or fewer loops," ASME J. Mechanical Design, 126:1:93-100, 2004. 
[13] G.S. Chirikjian and A.B. Kyatkin, Engineering Applications of Noncommutative Harmonic Analysis, CRC Press, 2001.

[14] A.J. Sommese, and C.W. Wampler, "Numerical Algebraic Geometry," Lectures in Applied Mathematics: The Mathematics of Numerical Analysis, Vol. 32, American Mathematical Society, Province, R.I., pp. 749-763, 1996.

[15] A.J. Sommese, J. Verschelde, and C.W. Wampler, "Advances in polynomial continuation for solving problems in kinematics," ASME J. Mechanical Design, 126:2:262-268, 2004.

[16] A.J. Sommese, and C.W. Wampler, Numerical Solution of Polynomial Systems Arising in Engineering and Science, World Scientific, 2005.

[17] A.J. Sommese, J. Verschelde, and C.W. Wampler, "Using monodromy to decompose solution sets of polynomial systems into irreducible components," In Application of Algebraic Geometry to Coding Theory, Physics, and Computation, ed. C. Ciliberto, et al., pp. 297-315, Kluwer Academic Publishers, 2001.

[18] A.J. Sommese, J. Verschelde, and C.W. Wampler, "Symmetric functions applied to decomposing solution sets of polynomial systems," SIAM J. Numerical Analysis, 40:6:2026-2046, 2002.

[19] T. Sasaki, "Approximate multivariate polynomial factorization based on zero-sum relations," Proceedings of the 2001 International Symposium on Symbolic and Algebraic Computation (ISSAC 2001), ed., B. Mourrain, pp. 284-291, ACM, 2001.

[20] D. Rupprecht, "Semi-numerical absolute factorization of polynomials with integer coefficients," J. Symbolic Comput., 37:5:557-574, 2004.

[21] Dixon, A.L., "The eliminant of three quantics in two independent variables," Proc. London Math. Soc., Ser. 2, 7:49-69, 1909.

[22] D. Cox, J. Little, and D. O'Shea, Ideals, Varieties, and Algorithms, Springer-Verlag, 1992.

[23] H.M. Möller and H.J. Stetter, "Multivariate polynomial equations with multiple zeros solved by matrix eigenproblems," Numerische Mathematik, 70:311-329, 1995.

[24] H.J. Stetter, Numerical polynomial algebra, Society for Industrial and Applied Mathematics (SIAM), Philadelphia, PA, 2004.

[25] S. Goedecher, "Remark on algorithms to find roots of polynomials," SIAM J. Sci. Comput., 15:5:1059-1063, 1994.

[26] C. Wampler, "Displacement analysis of spherical mechanisms having three or fewer loops," ASME J. Mechanical Design, 126:1:93-100, 2004. 
[27] H.-J. Su, C. Wampler, and J.M. McCarthy, "Geometric Design of Cylindric PRS Serial Chains," ASME J. Mechanical Design, 126:2:269-277, 2004.

[28] A.P. Morgan, "A transformation to avoid solutions at infinity for polynomial systems," Appl. Math. Comput., 18:77-86, 1986.

\section{Appendix A: Degree of $V_{N}$}

To analytically determine the degree of $V_{N}$, it is advantageous to modify Eq.(11) by introducing a new variable $\xi=\mathbf{q}^{T} \mathbf{q}$ and appending its defining equation. After homogenizing with the variable $w$, the new system is

$$
H_{N, k}(\xi, \mathbf{q}, \mathbf{t}, w)=\left[\begin{array}{c}
\mathbf{q}^{T} M_{i} \mathbf{q}+\xi \mathbf{n}_{i}^{T} \mathbf{t}, \quad i=1, \ldots, N \\
\mathbf{q}^{T} \mathbf{q}-\xi w \\
\mathbf{a} \cdot \mathbf{q}-w \\
A \mathbf{q}+B \mathbf{t}+C w
\end{array}\right]=0,
$$

where $M_{i}$ is as defined in Eq.(10). The trailing set of linear equations is size $k=6-N$, so we have in total 8 equations for the homogeneous variables $[\xi, \mathbf{q}, \mathbf{t}, w] \in \mathbb{P}^{8}$. The total degree is $2^{N+1}$. For all $N$, the point at infinity $[\xi, \mathbf{q}, \mathbf{t}, w]=[1,0,0,0]$ is a solution. For $N=1,2,3$ and general constraints and general linear slice, this point is nonsingular, as can be verified by forming the Jacobian matrix. This proves that the number of finite, isolated solutions is at most $2^{N+1}-1$ for $N=1,2,3$. The numerical results give this many roots, so the bound is seen to be sharp. For $N=4$, the situation is more difficult. The root $[1,0,0,0]$ is now singular and there is also a solution $[0,0$, null $B, 0]$. It is not easy to analytically determine the multiplicity of these points, but the numerical results show that the multiplicities to be two and 16, respectively, leaving 14 finite witness points.

For $N=5$, the solution $[\xi, \mathbf{q}, \mathbf{t}, w]=[0,0$, null $B, 0]$ is one-dimensional, so proper counting in the approach above becomes even more difficult. It is better to reformulate again, this time defining variables $\hat{\mathbf{t}}=\left(\mathbf{q}^{T} \mathbf{q}\right) \mathbf{t}$ so that the system becomes

$$
H_{N, k}(\xi, \mathbf{q}, \mathbf{t}, w)=\left[\begin{array}{cc}
\mathbf{q}^{T} M_{i} \mathbf{q}+\mathbf{n}_{i}^{T} \hat{\mathbf{t}}, & i=1, \ldots, 5 \\
\mathbf{a} \cdot \mathbf{q}-w \\
\left(\mathbf{q}^{T} \mathbf{q}\right) A \mathbf{q}+B \hat{\mathbf{t}}+\left(\mathbf{q}^{T} \mathbf{q}\right) C w
\end{array}\right]=0
$$

For $N=5, k=1$, the last row is a single hypersurface equation. Using Gaussian elimination, we may use the first three equations to express the elements of $\hat{\mathbf{t}}$ as quadratics in q. Substituting this to eliminate $\hat{\mathbf{t}}$ from the remaining equations, the remaining two point-plane constraints are quadratic while the final linear slice equation has become cubic. So the total degree of this reduced system is $2 \cdot 2 \cdot 3=12$. This proves an upper bound of 12 for the degree of $V_{5}$, and since the numerical results give 12 distinct, nonsingular witness points, we see that this bound is sharp. 


\section{List of Figures}

Figure 1. Schematic of locating six points on six planes.

Figure 2. Schematic of 3-2-1 locating.

Figure 3. Viewing a feature point $P$ with a pinhole camera.

Figure 4. Geometric constraints of the tripod mechanism. The triangle is free to move subject to each vertex remaining on a given plane.

Figure 5. Linear traces. Under parallel translation of the slicing line, the centroid of a witness set must follow a line. This is true for a composite set $\left(L_{0}\right)$ and for each of its irreducible pieces $\left(L_{1}, L_{2}\right)$.

\section{List of Tables}

Table 1. Degree of the motion variety $V_{N}=V\left(F_{1}, \ldots, F_{N}\right)$ for $N \leq 5$. 